respondents (EG) had a university degree more often ( $82 \%$ vs. $64 \%)$. The largest group of respondents were experienced patients with more than 10 years from diagnosis (47\% in Polish group (PG) and $54 \%$ in EG). The EG was more diversified in terms of a diagnosis.

The information about a disease and methods of treatment were the most important pieces of information for the respondents $(83 \%$ of PG rated it very important and $78 \%$ in EG) followed by finding a doctor ( $79 \%$ vs. $76 \%$ respectively). The three most important sources of information were: PG-Internet $46 \%$, rheumatologist 35\%, medical books 15\%; EG- Internet 43\%, rheumatologist $21 \%$, lectures for patients $-15 \%$.

Conclusions: The Results of Polish and English surveys were similar. The English group was more often interested in facilitating everyday life and finding support in living with a disease.

The test of knowledge on RMDs results confirmed that myths are still present among some patients. As much as $25 \%$ of Polish and $49 \%$ of international respondents associate occurrence of inflammatory rheumatic disease with weather conditions. Majority of people ( $94 \%$ in both groups) are aware that moderate physical activity is recommended in rheumatic diseases. The results also suggest insufficient knowledge about treatment methods and curability of RMDs It is important to increase efforts in dissemination of appropriate knowledge among patients and health professionals using different methods. Such attitude should help in minimizing adverse effects that result from lack of knowledge or self-awareness.

Acknowledgements: The study was financed from the statutory funds of the National Institute of Geriatrics, Rheumatology and Rehabilitation.

Disclosure of Interest: None declared

DOI: 10.1136/annrheumdis-2018-eular.1904

\section{PARE0014 GETTING THE PULSE ON WORKPLACE EXPERIENCES AND ACCOMMODATIONS}

L. Proulx ${ }^{1}$, L. Proulx ${ }^{1}$, N. Robertson ${ }^{1}$, D.P. Richards ${ }^{2}$, L. Wilhelm ${ }^{3}$, J. Gunderson ${ }^{4}$ A. Sirois ${ }^{5}$, A. McKinnon $2 .{ }^{1}$ Canadian Arthritis Patient Alliance, Ottawa; ${ }^{2}$ Canadian Arthritis Patient Alliance, Toronto; ${ }^{3}$ Canadian Arthritis Patient Alliance, King's County; ${ }^{4}$ Canadian Arthritis Patient Alliance, Glaslyn; ${ }^{5}$ Canadian Arthritis Patient Alliance, Montreal, Canada

Background: As a patient-driven and managed non-profit organization, the Board members of the Canadian Arthritis Patient Alliance (CAPA) have experienced many of the workplace challenges of arthritis first-hand. Recognizing the importance of being able to contribute in the workplace, CAPA recently initiated a project focused on broadening the understanding of the challenges faced by people living with arthritis in the workplace; and, to develop workplace tools and appropriate advocacy activities to address these challenges.

Objectives: A survey concerning arthritis in the workplace was developed with the goal of enhancing the understanding of the workplace challenges faced by people with arthritis, including the type and effectiveness of personal and workplace accommodations and the effectiveness of available resources.

Methods: A Board member/project manager developed the survey in consultation with people living with arthritis. The survey was available in English and French and targeted to people who live with various forms of arthritis, including inflammatory arthritis as well as Systemic Lupus Erythematosus, osteoarthritis, etc. The survey was distributed to membership in December 2017 through the newsletter and social media channels. Further promotion of the survey was completed through outreach to French and English patient and non-profit groups. The results were collected online through a survey platform and analyzed using basic statistical techniques.

Results: At the date of submission, 218 survey responses were received (127 English responses, 91 French responses) and the majority of respondents(over $90 \%$ ) live in Canada. Over $80 \%$ of respondents live with inflammatory arthritis and $86 \%$ reported that arthritis affected them moderately or significantly in the workplace. Although $49 \%$ of respondents indicated that working had a positive effect on their lives, $66 \%$ indicated that working took energy away from other life activities. The most highly helpful workplace accommodations were: flexible hours of work (58\%), breaks to give joints/body time to recover (55\%), paid time away from work to attend medical appointments (47\%), and working from home one or more days per week (39\%). The most highly used personal accommodations were: reduction of social activities (77\%), pacing during the work day $(70 \%)$ and spreading out non-work activities (64\%). The survey responses will be further summarized for the presentation, along with ideas for development of resources based on the gaps identified.

Conclusions: CAPA developed a workplace survey to broaden the understanding of the challenges faced by people living with arthritis in the workplace. Early analysis of the survey indicates a number of implications such as the significant impact of arthritis on the workplace and the impact on other life activities. CAPA will develop appropriate workplace tools and plan advocacy activities in order to address the survey findings.
Acknowledgements: CAPA would like to thank the organizations that collaborate with us as well as various pharmaceutical companies whose funding enables our operation.

Disclosure of Interest: L. Proulx Grant/research support from: CAPA receives unrestricted grants from a variety of pharmaceutical companies, Speakers bureau: provided ad hoc speaking to Sanofi Genzyme, L. Proulx: None declared, N. Robertson: None declared, D. P. Richards: None declared, L. Wilhelm: None declared, J. Gunderson: None declared, A. Sirois: None declared, A. McKinnon: None declared DOI: 10.1136/annrheumdis-2018-eular.4041

\section{PARE0015 LARGE-SCALE SURVEY OFFERS GENERAL INSIGHT AND BASIS FOR POLICY DRAFTING}

S. De Jong, I. Koopmans, M. Feith. Patient advocacy, Dutch Arthritis Foundation, Amsterdam, Netherlands

Background: The Dutch Arthritis Foundation (DAF) commissioned a large-scale survey to gain a broad insight into the various aspects of living with RMDs in the Netherlands. The results offer an insight into the prevalence of RMDs, as well as into living with RMDs and the use and standard of relevant healthcare and socia care in our country. This was the first survey of this scale in the Netherlands.

Objectives: The survey aimed to generate data about the prevalence and the impact of RMDs on people's every-day lives in the Netherlands. The DAF will use the results to better inform parties involved in caring for people with RMDs and to help them with policy drafting, such as authorities, healthcare insurers and health care providers.

Methods: The bulk of all data consists of statistical data from the NIVEL Health care Registrations (from 355 GP clinics, which register 1.1 million RMD patients) The survey also built on statistical and qualitative data from Vektis (business intelligence centre for the healthcare industry) and the National Institute of Public Health and Environmental Protection. Patients were contacted through the National Panel for Chronical Patients and asked to score their quality of life, the impact RMD has on their lives, and the standard of care. Rheumatologists, health professionals in rheumatology and orthopaedics were also represented in the monitoring group. The survey was carried out for four key disorders - chronic inflammatory rheumatism, osteoarthritis, gout and osteoporosis. These disorders are registered by both GPs and specialists. Fibromyalgia is not registered as a specific disorder by GPs and was therefore outside the scope of the survey.

Results: The report generated the following results for the more than two million people with RMDs in the Netherlands:

1 in 9 people have a RMD; More than 2 million people have a RMD; Every day, 700 people are diagnosed with a form of RMD; There are 1.3 million people with osteoarthritis; 400,000 people with osteoporosis; 370,000 people with gout; 220,000 people with inflammatory rheumatism such as RA, ax-SpA, SLE, Sjögren syndrome and scleroderma.

$69 \%$ of people with RMD are restricted by their symptoms in their day to day life;

$50 \%$ of people with RMD between the ages of 40 and 65 are not in paid employment;

$54 \%$ of people with RMD indicate they suffer from loneliness;

$50 \%$ of people with RMD deal with incomprehension;

$65 \%$ uses medication, $37 \%$ physiotherapy.

Conclusions: The report shows the extent of the impact of RMDs, not only medically, but also with regard to social effects. This was the first study to adequately reveal the sense of loneliness among RMD patients, laying bare the incomprehension they face in others. It also studied the impact of RMDs on employment, the great reliance on voluntary carers, and the fact that many people with a RMD also suffer other symptoms. The report was able to pinpoint the most vulnerable patients, and therefore provides a clear indication as to where improvements can be made. It also clearly shows that there is room for improvement when it comes to the registration of specific disorders such as fibromyalgia, but also disorders classed as a form of inflammatory arthritis, to enable the generation of objective data from the registration systems.

Disclosure of Interest: None declared

DOI: 10.1136/annrheumdis-2018-eular.5290

\section{PARE0016 THE ROLE OF EMPATHY QUOTIENT IN PATIENT- PHYSICIAN COMMUNICATION: A TOOL TO IMPROVE MEDICATION ADHERENCE}

A. Celano. Italian National Association for People with Rare and Rheumatic Diseases - APMAR Italia, Lecce, Italy

Background: Brain circuits governing the identification with others and those regulating the care feeling towards suffering people form wide distinct brain networks and are essential elements of empathy. This last is ruled by the "circuit of 